\title{
THE ROLE OF SHAPING IN ACHIEVING HIGH PERFORMANCE IN DIII-D
}

by

E.A. LAZARUS, ${ }^{*}$ A.W. HYATT, T.H. OSBORNE, S.E. ATTENBERGER, ${ }^{*}$ M.E. AUSTIN, ${ }^{\dagger}$ K.H. BURRELL, T.A. CASPER, ${ }^{\ddagger}$ M.S. CHU, J.W. CUTHBERTSON, ${ }^{\diamond}$ E.J. DOYLE, ${ }^{\nabla}$ P. GOHIL, J.M. GREENE, C.M. GREENFIELD, R.J. GROEBNER, W.A. HOULBERG, ${ }^{\star}$ C.-L. HSIEH, G.L. JACKSON, J. KIM,

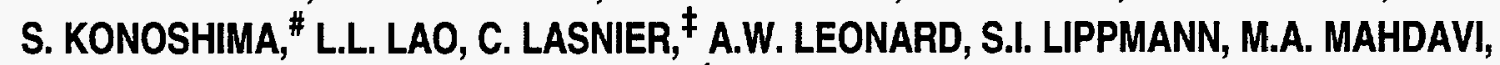
R. MAINGI, ${ }^{\star}$ R.A. MOYER, ${ }^{\nabla}$ C.L. RETTIG, ${ }^{\ddagger}$ B.W. RICE, ${ }^{\ddagger}$ M.J. SCHAFFER, D.P. SCHISSEL, R.T. SNIDER, R.D. STAMBAUGH, R.E. STOCKDALE, T.S. TAYLOR, A.D. TURNBULL, M.R. WADE, ${ }^{*}$ R. WOOD, ${ }^{\ddagger}$ and D. WROBLEWSKI ${ }^{\ddagger}$

This is a preprint of a paper to be presented at the Fifteenth International Conference on Plasma Physics and Controlled Nuclear Fusion, September 26 through October 1, 1994, in Madrid, Spain, and to be published in the Proceedings.

Work supported by

U.S. Department of Energy

Contract Nos. DE-AC03-89ER51114, DE-AC05-OR21400, W-7405-ENG-48, DE-AC04-94AL85000, DE-FG03-89ER51121, and DE-FG03-86ER53225

*Oak Ridge National Laboratory.

†niversity of Maryland.

† Lawrence Livermore National Laboratory
Sandia National Laboratory at Livermore

$\checkmark$ University of California at Los Angeles

\# Japan Atomic Energy Agency

\section{GENERAL ATOMICS PROJECT 3466} OCTOBER 1994 


\section{DISCLAIMER}

This report was prepared as an account of work sponsored by an agency of the United States Government. Neither the United States Government nor any agency thereof, nor any of their employees, make any warranty, express or implied, or assumes any legal liability or responsibility for the accuracy, completeness, or usefulness of any information, apparatus, product, or process disciosed, or represents that its use would not infringe privately owned rights. Reference herein to any specific commercial product, process, or service by trade name, trademark, manufacturer, or otherwise does not necessarily constitute or imply its endorsement, recommendation, or favoring by the United States Government or any agency thereof. The views and opinions of authors expressed herein do not necessarily state or reflect those of the United States Government or any agency thereof. 


\section{DISCLAIMER}

Portions of this document may be illegible in electronic image products. Images are produced from the best available original document. 
IAEA-CN-60/A-5-I-I

\title{
THE ROLE OF SHAPING IN ACHIEVING HIGH PERFORMANCE IN DIII-D
}

\begin{abstract}
Experiments to study the effect of plasma shape on performance show that the high performance levels characteristic of $\mathrm{VH}$-mode plasmas are only obtained for high triangularity $(\delta)$. A characteristic difference in central heating efficiency is observed when high and low $\delta$ plasmas are compared. We find this difference to correlate well with the attainment of Mercier stability. The core plasma for the high $\delta$ cases achieves Mercier stability when $q_{0}$ rises above a critical value which depends on $\delta$, providing direct access to the second-stable regime. Other attributes of the VH-mode commence after a delay. The rise in $q_{0}$ is attributable to the radial gradients in the neutral beam and bootstrap current sources. Simulation indicates that such plasmas would persist for many seconds if the rise in density could be controlled, maintaining a centrally peaked neutral beam deposition profile.
\end{abstract}

\section{INTRODUCTION}

In 1993 DIII-D mounted a major campaign to study the effect of shape on plasma performance. A primary motivation for the investigation is to determine what plasma shape will be compatible with the DIII-D Radiative Divertor [1] and Advanced Tokamak [2] Programs planned for the next several years. In part the effort was motivated by previous observation that performance was superior in double-null as compared to single-null plasmas [3]. There remained an ambiguity as to whether "nullness" (1 or 2$)$ or plasma shape was the critical variable. This work resolves that ambiguity in favor of shape, and provides further insight into the effects of shaping. While it is expected that the beta limit will depend on shape, we also find that confinement, at finite beta, depends on the plasma shape. We further find that the plasma triangularity is most important to performance while plasma elongation plays a weaker role.

The product $\beta \cdot \tau_{E}$ has been used as a figure-of-merit for comparing the fusion gain of different toroidal magnetic configurations [4]. Here we shall use it as the figure-of-merit for comparing different configurations within the DIII-D tokamak. We find the plasma shape to be a dominant consideration. In previous work [5] on the optimization of $\beta$ we introduced a parameter describing plasma shape,

$$
\mathcal{S} \equiv\left(\frac{I_{p}}{a B_{t}}\right) \cdot q_{\psi}
$$

Our motivation was that the beta limit should be the intersection of the $n=1$ and $\mathrm{n}=0$ stability boundaries, and that $\mathcal{S}$, or more properly, $\mathcal{S} \ell_{i}$, is bounded by $\mathrm{n}=0$ stability. Then the familiar Troyon limit [6] can be expressed as $\beta_{T}^{m a x} \approx 4 \frac{\mathcal{S} \ell_{i}}{q_{\psi}}$, i.e., in terms of limits to $n=0$ and $n=1$ stability. It is somewhat surprising that confinement is also affected by plasma geometry, and we continue to use the descriptor $\mathcal{S}$. For diverted plasmas we substitute $q_{95}$ for the boundary 
value $q_{\psi}$. We avoid description of performance in terms of $\kappa$ and $\delta$ since higher moments of the shaping field appear to be important and the interaction of $\kappa$ and $\delta$ in determining plasma performance is complex. Similarly, the strong effect of the safety factor on plasma confinement makes it difficult to describe the experiment in terms of $I_{p}$, the plasma current. $S$ is a measure of the toroidallyweighted poloidal contour length. We note in passing that the values of $\mathcal{S}$ in the experiments reported here do not represent plasmas for which there is any difficulty with control of the axisymmetric stability on DIII-D [7] and the best plasmas have a stability margin of 1.4.

In this paper we will address three issues: 1) global results on the parametric dependencies of $\beta \cdot \tau_{E}, 2$ ) the time evolution of the profiles, particularly temperature, for differing plasma shapes and the dynamics of the current profile evolution, and 3) the stability of the high-pressure plasma core.

In presenting global results, it will appear that we are discussing a continuum in the quality of the discharge. To the extent that shaping merely affects the ratio of plasma current to safety factor, this appears to be true. However, when we examine the plasma core, the results appear more as a bifurcation between those plasmas which attain Mercier stability and those which do not. The high performance phase is transient, and, in fact, a VH-mode [8] which only occurs for the higher triangularity. The low triangularty plasmas remain $\mathrm{H}$-mode. This paper will focus on the core plasma, which we find to be second-stable. A thorough discussion of VH-mode conditions existing near the boundary and the role of $\vec{E} \times \vec{B}$ velocity shear in stabilization in the region $\sqrt{\tilde{\psi}} \gtrsim 0.8$ may be found in [9]. We remind the reader that in considering the Mercier criterion for shaped plasmas both $\kappa$ and central $\beta$ are destabilizing while triangularity is strongly stabilizing.

The experiment focussed on the four shapes identified in Table I; high and low elongation $(\kappa)$ at high and low triangularity $(\delta)$. Although Shape $A$, the standard DIII-D double-null, has been studied extensively, the addition of carbon armor tile to the outer wall made it necessary that we reestablish a reference for the other shapes. Indeed, there was some improvement in plasma performance with the additional armor, which will be discussed. Although the vessel was boronized for these experiments, $\mathrm{VH}$-mode plasmas were observed, with this new armor tile, without boronization. The most visible change resulting from this additional armor was a reduction in metallic impurities. Typical values of selected parameters for each shape are shown in Table I.

Table I. Typical Values of Selected Parameters for Each Shape

\begin{tabular}{cccccc}
\hline & & & \multicolumn{1}{c}{$I_{p}$ (MA) } \\
Shape & $\mathcal{S}$ & $\kappa$ & $\delta$ & at $q_{95}=3$ & $\mathcal{S}^{2} R_{0}^{2} / 1+\kappa^{2}$ \\
\hline A & 6.54 & 2.1 & 0.85 & 2.9 & 22.2 \\
B & 5.07 & 1.7 & 0.85 & 2.2 & 17.7 \\
C & 5.15 & 2.1 & 0.32 & 2.2 & 13.5 \\
D & 3.84 & 1.8 & 0.30 & 1.7 & 10.1 \\
\hline
\end{tabular}

For each shape, heating with neutral beams was varied from 5 to $15 \mathrm{MW}$, and $q_{95}$ was varied over the range 3.2 to 5.6 . The toroidal magnetic field $B_{t}$ was 
fixed at $2.1 \mathrm{~T}$, as previous work [3] indicated that highest $\beta \cdot \tau_{E}$ values occur at the highest toroidal field. The procedure was to find a minimum target density, and begin neutral beam injection about $0.4 \mathrm{~s}$ after plasma current reached flat top. The plasmas with best shape exhibit a VH-mode phase which terminates with a global instability [10], whereupon it reverts to an ELMing $\mathrm{H}$-mode. A more detailed discussion of the ELMing phase of the discharge is contained in Ref. [11]; briefly, enhancement of $\beta \cdot \tau_{E}$ beyond the expected $I_{p}$ scaling vanishes in the ELMing $\mathrm{H}$-mode. The principal diagnostics are Thomson scattering $\left(T_{e}\right.$, $n_{e}$ ) charge exchange recombination (CER) spectroscopy $\left(T_{i}\right)$, electron cyclotron emission (ECE) (central $T_{e}$ ) and motional Stark effect (MSE) (q). Of particular note are improvements in the MSE diagnostic by using optics exhibiting a muchreduced Faraday effect, which has reduced the uncertainty in the reconstructed q-profile.

In this paper, $W$ is the total stored energy, $W_{f}$ is the energy stored in fast ions, $P$ is the total input power, $\tau \equiv \mathrm{W} / \mathrm{P}, \tau_{E} \equiv W /(P-\dot{W})$, and $\tau_{E}^{\text {th }} \equiv$ $\left(W-W_{f}\right) /(P-\dot{W})$. If Eq. (1) is combined with approximate DIII-D/JET scaling [12] allowing a multiplicative factor $F$,

$$
\tau_{E}=F \cdot \tau_{E}^{D J} \approx F \cdot 0.11 \frac{I_{p} R^{\frac{3}{2}}}{\sqrt{P}} .
$$

If we express plasma volume as $2 \pi R\left[\pi \bar{a}^{2}\right]$, where $\bar{a}=a \sqrt{\frac{1+\kappa^{2}}{2}}$ then Eqs. (1) and (2) combine in a simple expression for $\beta \cdot \tau_{E}$,

$$
\beta \cdot \tau_{E}=0.31\left(\frac{\mathcal{S}^{2} R^{2}}{1+\kappa^{2}}\right)\left(\frac{F}{q}\right)^{2},
$$

which separates into geometric, $\mathcal{S}^{2} R^{2} /\left(1+\kappa^{2}\right)$, and confinement, $(F / q)^{2}$, factors. We previously [3] used $V=2 \pi R\left[\pi \kappa a^{2}\right]$, but this experssion better resolves shapes $\mathrm{B}$ and $\mathrm{C}$, and is an equally good approximation to the actual volume of the plasma.

Section 2 reports on the global results of this study, Section 3 on profile evolution, Section 4 on stability analysis, and Section 5 summarizes the work.

\section{GLOBAL RESULTS}

\subsection{Discharge Evolution}

A characteristic of $\mathrm{H}$-mode plasmas is the rise in density during the ELMfree phase. Minimizing this density rise favorably affects plasma performance: a key to high performance in DIII-D is wall conditioning to minimize the density rise. Preparation includes boronization, preceded and followed by high temperature bakes $\left(350^{\circ} \mathrm{C}\right)$ done every few weeks of operation and between-shot helium glow at an electrode voltage above $300 \mathrm{~V}$. The signatures seen during operation are a low level of recycle, as evidenced by minimal rise in the divertor $D_{\alpha}$ light during the ELM-free phase, and low $\left(\approx 10^{-6}\right.$ torr $)$ neutral pressure during the discharge as measured at the outside midplane [13]. As will be discussed, the achievement of these conditions in low $\delta$ shapes will not produce high-performance plasmas. 
The time evolution of a representative plasma for shapes $A$ (best) and D (worst) are shown in Fig. 1. In each case the current ramp was held at less than $1 \mathrm{MA} / \mathrm{s}$, part of the prescription for avoiding locked modes. The density was programmed to avoid locked modes $\left(q^{* 2} n_{e} R_{0} / B_{t}>72 \times 10^{19} \mathrm{~m}^{-2} \mathrm{~T}^{-1}\right)$, and usually raised somewhat above this limit to achieve best performance. Neutral beams were injected about $400 \mathrm{~ms}$ after the current reached its programmed value.

For shapes $\mathrm{A}$ and $\mathrm{B}$, as soon as beam heating begins $\beta, \beta \cdot \tau_{E}, q_{0}, \bar{n}_{e}$, and central temperatures all rise rapidly. For this particular shot, 78136 , central $T_{i}$ exceeded $20 \mathrm{keV}$ and, typically, in the VH-phase $T_{i}$ is 2-3 times $T_{e} . T_{i}(0)$ peaked at $2290 \mathrm{~ms}$ and then dropped precipitously to about $15 \mathrm{keV}$ and remained there until the end of the VH phase at $2430 \mathrm{~ms}$. After regular ELMing begins $T_{i} \approx T_{e}$. If the target density is slightly higher than in this case, the $T_{i}(0)$ does not reach such a high value, but somewhat higher $\beta \cdot \tau_{E}$ is achieved. In the VH-phase virtually all the beam power is deposited in the ion channel. Because of the rise in density the deposition profile is broadening continuously from the start of heating. As predicted in Eq. (3), the power dependence of $\beta \cdot \tau_{E}$ is weak, with higher power resulting in higher beta at reduced confinement. Typically $W_{f}$ is $10 \%$ of $W$ in these plasmas. Even in 78136 at $2290 \mathrm{~ms} W=2.5 \mathrm{MJ}$ and $W_{f}=0.4 \mathrm{MJ}$. The $\beta$ and $q_{0}$ values here are from equilibrium reconstructions at $10 \mathrm{~ms}$ intervals.

There are characteristic differences in the temporal behavior of shapes $\mathrm{A}$ and $\mathrm{B}$ as compared to $\mathrm{C}$ and $\mathrm{D}$ during the ELM-free period. The high triangularity plasmas are characterized by a cessation of sawtoothing along with a rapid rise in $\beta$ and central temperature, whereas the low triangularity plasmas continue sawtoothing and show a more modest rise in these parameters. It is clear that in shape A $q_{0}$ rises above unity at $2050 \mathrm{~ms}$, well before the "spin-up" [9] time $(2190 \mathrm{~ms})$ when velocity shear apparently stabilizes fluctuations near the $q=2$ surface. While we speak of an ELM- and sawtooth-free phase, it is almost always the case that we observe an occasional ELM and/or sawtooth-like event during this interval; it is better described as virtually free of ELMs and sawteeth with neither correlated with a global loss of confinement.

For shapes A [Fig. 1(a)] and B, $\beta$ and $\tau_{E}$ rise continuously until this phase is terminated by a global instability, whereupon the plasma reverts to an ELMing, sawtoothing $\mathrm{H}$-mode at $2430 \mathrm{~ms}$.

In comparing $A$ and $B$, we note a marked difference in the duration of the ELM-free phase. For shape B, in no case did the ELM-free duration exceed $250 \mathrm{~ms}$, while in shape A durations up to $1300 \mathrm{~ms}$ were observed. While similar values of $\beta_{N} \cdot F$ were achieved, the evolution was much more rapid for shape B.

Shot 79716 [Fig. 1(b)] is representative of the lower triangularity plasmas. There is no VH phase, and the plasma is typical of DIII-D H-modes. Note that $q_{0}$ does not rise above unity. In this paper we will avoid for the most part the distinction between $q_{0}=1$ and $q_{0}=q_{m} \approx 1$ where the Mercier criterion is satisfied. Additionally, it is not clear that the Mercier criterion is ever violated, in that the low $\delta$ cases we have observed have slightly positive central $p^{\prime}$.

\subsection{Global Behavior}

In the course of the experiment, for each discharge a number of times of interest (e.g., peak $\beta$, peak $\beta \cdot \tau_{E}, \mathrm{VH}$-termination, etc.) were identified and a data base was formed. Results from the analysis of this database are now presented. Motivated by Eq. (3), we plot $\beta \tau_{E}$ vs. the quantity $\mathcal{S}^{2} R^{2} /\left(1+\kappa^{2}\right)$ 

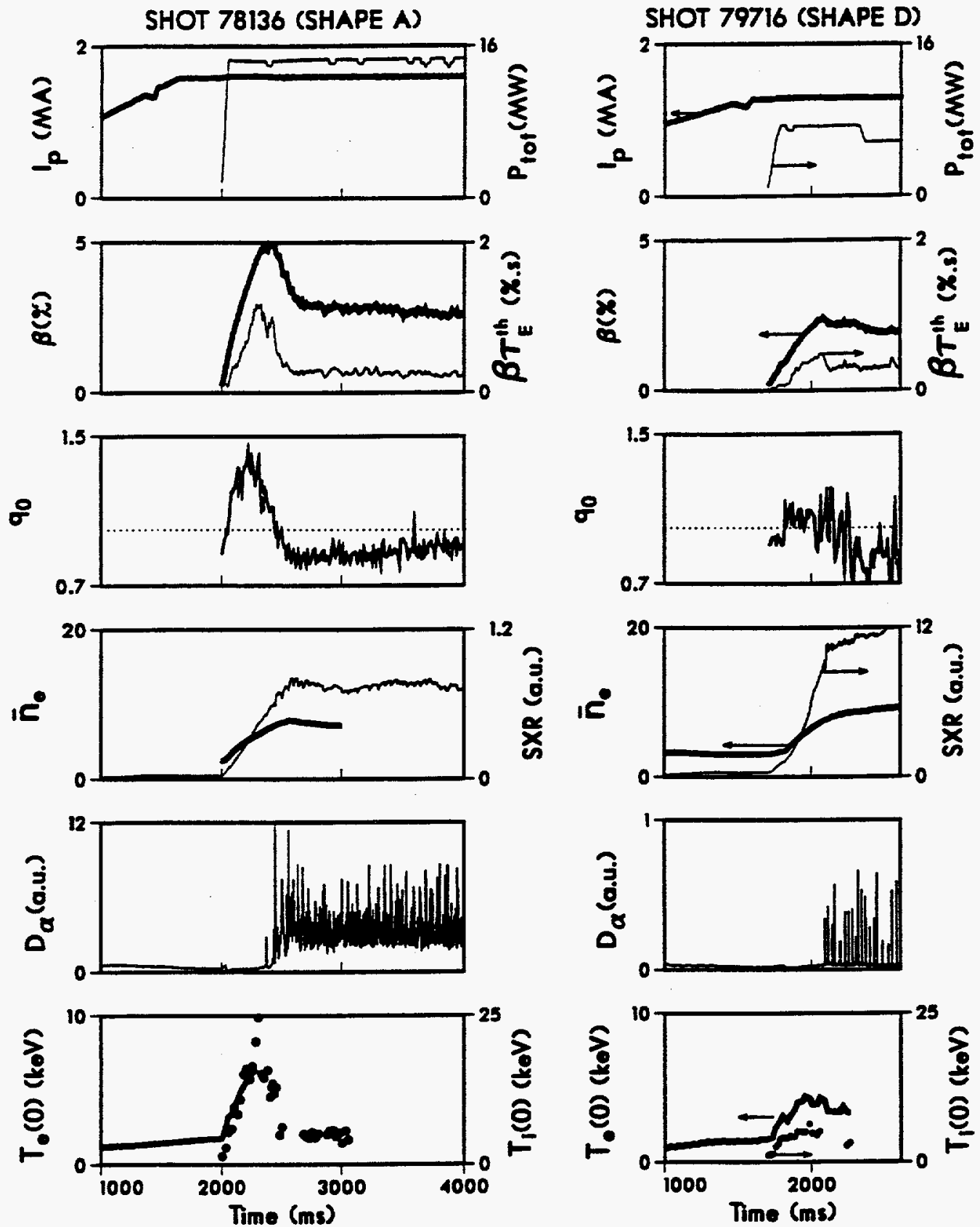

Fig. 1. Typical disharge evolution for high and low $\delta$ plasmas. The righthand scale applies to the thinner line. (a) Shot 78136, a hot-ion VH-mode, and (b) a low $\delta$ plasma showing typical ELMing H-mode behavior.

in Fig. 2. Each datum is the maximum value for a single discharge with the constraint $\dot{W} / P_{\text {tot }} \leq 0.33$. One sees a clear trend of improved performance with the geometric factor. This figure differs somewhat from Fig. 3 of Ref. [3] in that $\dot{W}$ corrections are included here, there is only a single time point per discharge 


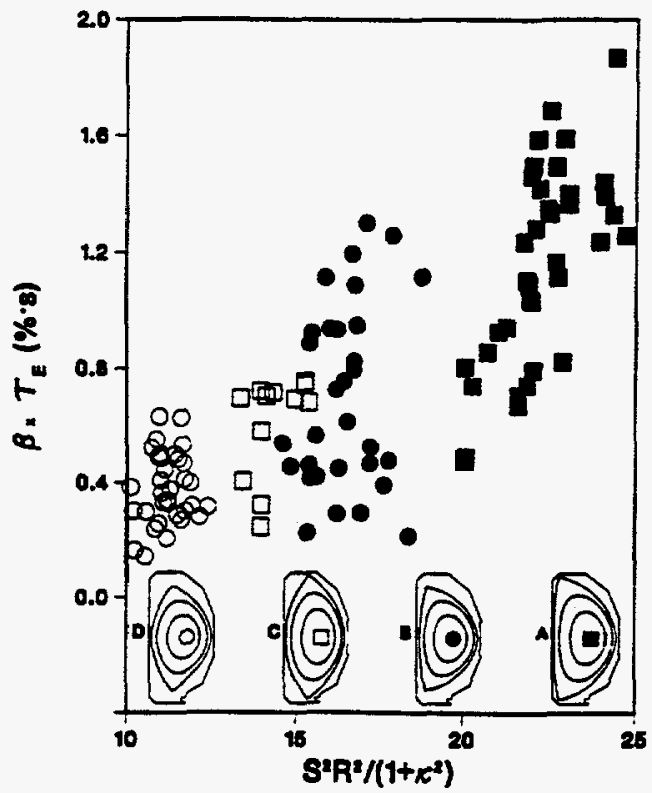

Fig. 2. $\beta \cdot \tau_{E}(\% . s)$ vs. $\mathcal{S}^{2} R^{2} /\left(1+\kappa^{2}\right)$ (m). The four shapes are also shown, with the symbol which will be used to identify shapes in subsequent figures.

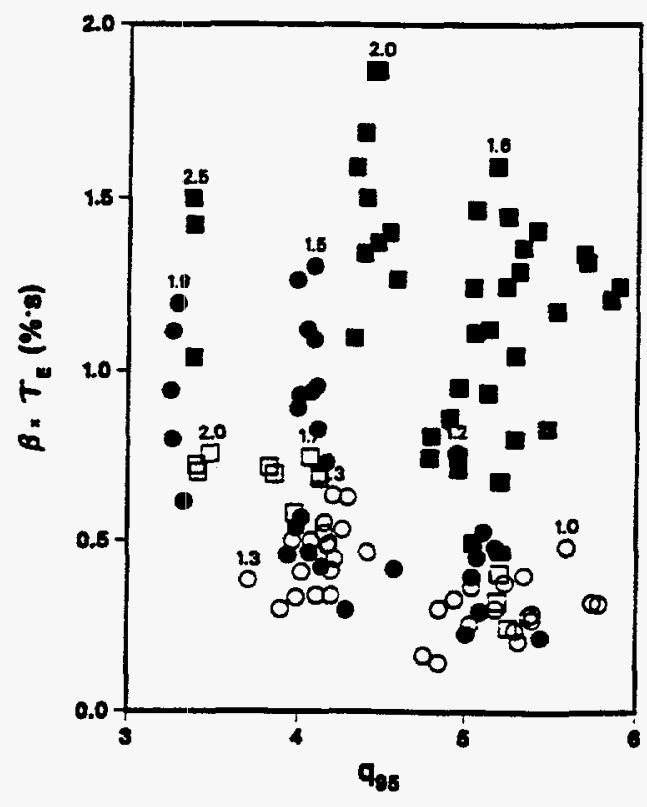

Fig. 3. $\beta \cdot \tau_{E}$ (\%.s) vs. $q_{95}$. Selected points are labeled with $I_{p}(M A)$.

similar to the earlier work, and demonstrate that the principal dependence is the plasma geometry, and not whether the divertor is single or double-null. The data sets are mutually exclusive.

The reader may notice that there are more entries for our standard DN shape (A) than the other shapes. Whenever there was doubt about the tokamak conditions or difficulty in obtaining good performance in the other shapes we would return to this shape to benchmark the tokamak. In fact an enormous effort was made, particularly on shape $D$, to obtain good performance. Contrary to the experience with shapes $A$ and $B$, improved tokamak conditions and operation did not result in any improvement in performance, although the ELM-free duration was lengthened to about $200 \mathrm{~ms}$. Also, raising triangularity from 0.3 to 0.55 had no observable affect on performance at this elongation.

The variation within each group is the result of a number of factors: target density, safety factor, beam power, shot-to-shot variation in the level of MHD activity, etc. However, there are unexplained subtleties such as X-point location. Moving the outer strike points closer to the wall will increase the ELM-free duration well beyond the effect of changing shape. This longer ELM-free period will result in higher $\beta \cdot \tau_{E}$. However, the most significant variation appears to be the result of differences in safety factor. In Fig. 3 we plot this same data set with $q_{95}$ as the abcissa. For shapes A and B, $\beta \cdot \tau_{E}$ peaks at $q_{95} \gtrsim 4$, again consistent with our earlier work. For selected points we have noted the plasma current (in MA) just above the datum. One can readily see why we avoid trying to portray the results in terms of $I_{p}^{2}$. The $\mathcal{S}$ and $q_{95}$ dependencies are much stronger than any direct scailing with $I_{p}^{2}$. For the lower triangularity shapes there does not seem to be a clear dependence within this data set. 
In plotting $\beta \cdot \tau_{E}$, we avoid obscuring the absolute level of performance with assumed scalings which are clealy limited in their scope. Nevertheless, we still want to quantify how much of the improvement in $\beta \cdot \tau_{E}$ represents an enhancement over $I_{p}^{2}$ scaling. In Fig. 4 we plot $F$ from (2) vs. $\beta_{N}$. In this figure we add the DIII-D "confinement" database to the database of this experiment. In this way, we can say that we include any results of note which have been achieved on DIII-D. These points, shown as smaller symbols, are sorted by shape and restricted in $\beta \cdot \tau_{E}$ as indicated on the figure. The dashed lines represent the rough boundaries for the high and low triangularity shapes, namely, $\beta_{N} F=9.0$ and 6.2 respectively. For plasmas of in this figure, the relation to ITER-P scaling [14] with its $H$-factor, also shown, is $H=1.35 F+0.16$ to within a few percent accuracy. Thus for the best plasmas there is about a $50 \%$ improvement in performance with plasma shape beyond that expected based on $I_{p}^{2}$ scaling. A characteristic difference seems to be that the onset of degradation of confinement occurs at higher $\beta_{N}$ for higher triangularity plasmas.

From these results we conclude that plasma performance depends more strongly on triangularity than on elongation. Note in Fig. 2 how much worse shape $C(\delta=2.1)$ is than either shape A or B. At a fixed triangularity elongation is beneficial in that it allows higher $I_{p} / q$.

Now we examine the confinement factor in Eq. $(3),(F / q)^{2}$. In Fig. 5 we plot $F$ vs. $q_{95}$. Here we have combined databases as in the previous figure.

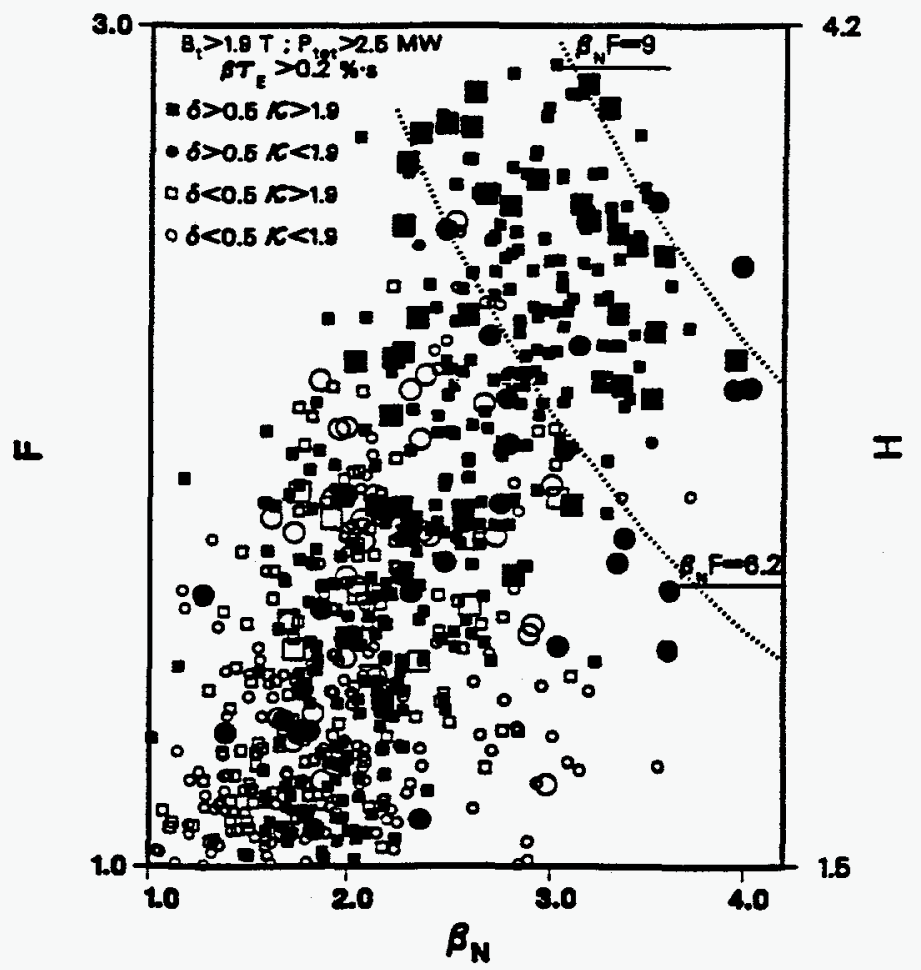

Fig. 4. $\beta_{N}$ vs $F, \beta_{N} \equiv \beta /\left(I_{p} / a B_{t}\right.$ and $F$ is the enhancement over DIII-D/JET confinement scaling. The dashed lines are $\beta_{N} F=6.2$ and 9 . The small symbols are from a separate data base containing a greater variety of plasma shapes. $H$ on the right hand scale is approximated using $H=1.35 F+0.16$. 


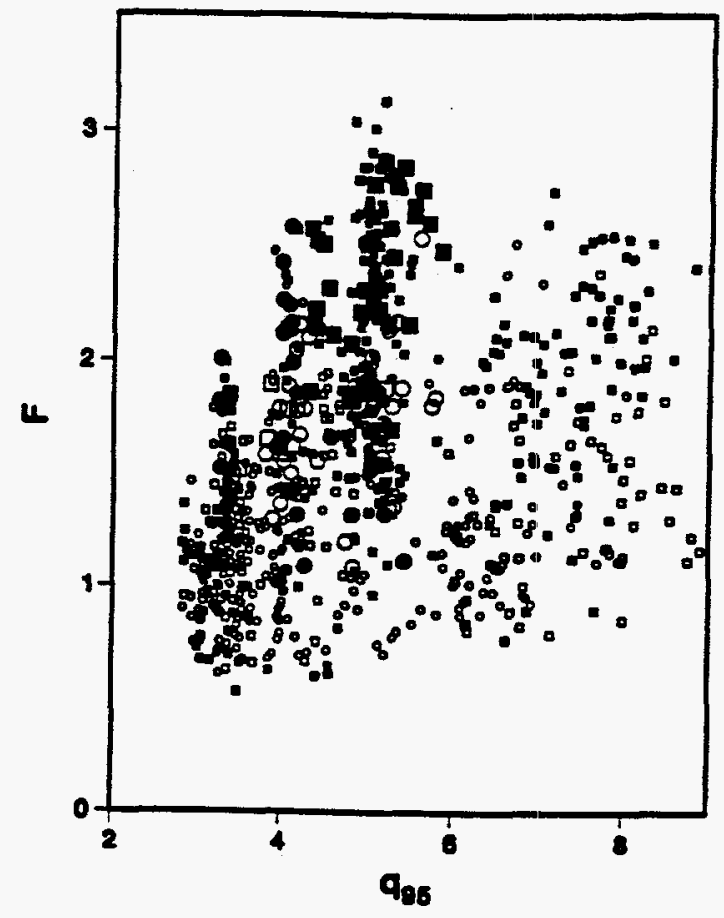

Fig. 5. $F$ vs. $q_{95}$. At $q_{95}<4$ the degradation of confinement is greater than the increase in $\beta$, leading to an overall decrease in $\beta \cdot \tau_{E}$. Also, $F$ decreases at At $q_{95}>6$. This result is consistent with other DIII-D H-mode plasmas.

There is a clear peak in $F$ at $q_{95}$ about 5 , and for lower $q_{95}, F$ plummets. Also, for higher triangularity higher values of $F$ are observed. Here we clearly see the penalty of lower $q_{95}$ operation. Summarizing, $\beta_{N} F$ peaks at $q_{95} \gtrsim 5$ and $\beta_{t} \tau_{E}$ at $q_{95} \gtrsim 4$. The results are quite consistent with a previous comparison of singleand double-null diverted plasmas. There is as much as a $50 \%$ improvement in performance beyond that predicted by $I_{p}^{2}$ scaling which is the topic of the remainder of this paper.

\section{PROFILE CHARACTERISTICS}

The higher performance in the high $\delta$ plasma is readily seen in the evolution of the kinetic profiles as well as the global results. In generating profiles for DIII-D data it is necessary to map all the data into a flux coordinate. In this paper we will virtually always use $\sqrt{\tilde{\psi}}, \tilde{\psi}$ being normalized poloidal flux. The equilibrium used for this reconstruction is generated using the magnetics data and 7 or $8 \mathrm{MSE}$ channels to constrain $p^{\prime}$ and $f f^{\prime}$. Equilibria on which stability analysis is performed have been through an additional cycle where the plasma pressure is calculated from the profile data and a standard calculation of $W_{f}$ and then used in a second equilibrium fit to provide more accurate internal profiles $\left(p^{\prime}\right.$ and $\left.f f^{\prime}\right)$.

In Figs. 6 and 7 we compare the profile evolution for the first few hundred $\mathrm{ms}$ in of neutral beam heating for shapes $\mathrm{A}$ and $\mathrm{C}$. The particular choices (Shots 79604 and 80072) are chosen because they have the same plasma current (2 MA) 

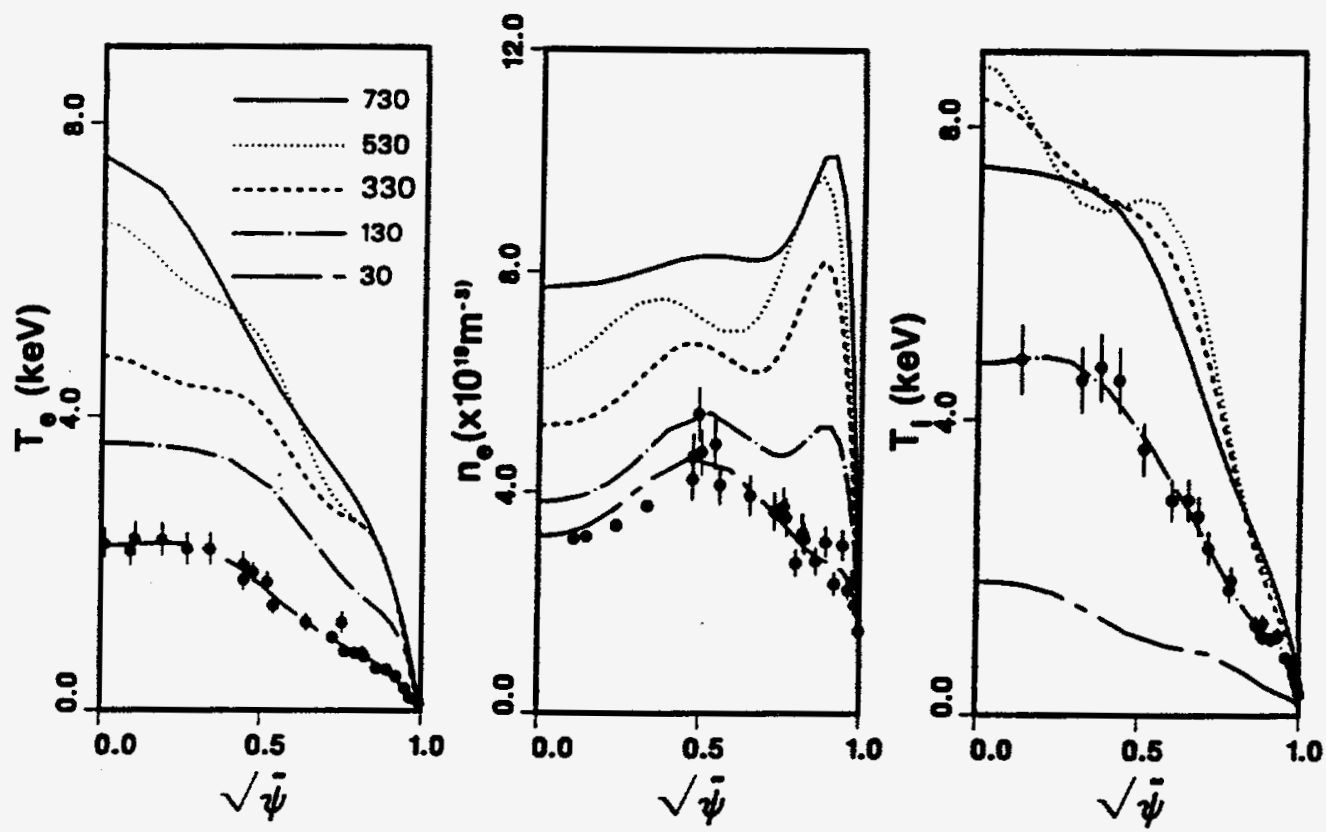

Fig. 6. Temperature and density profile evolution in a VH-mode plasma vs. $\sqrt{\bar{\psi}}$. This shot reached the record value for $\beta \cdot \tau_{E}$, but not for $n_{D}(0) T_{i}(0) \tau_{E}$. Shot 79604, $I_{p}=2 M A, P_{b}=11 \mathrm{MW}, S H A P E A, q_{95}=4.9$. Times labeled are in $m s$, relative to the start of beam-heating.
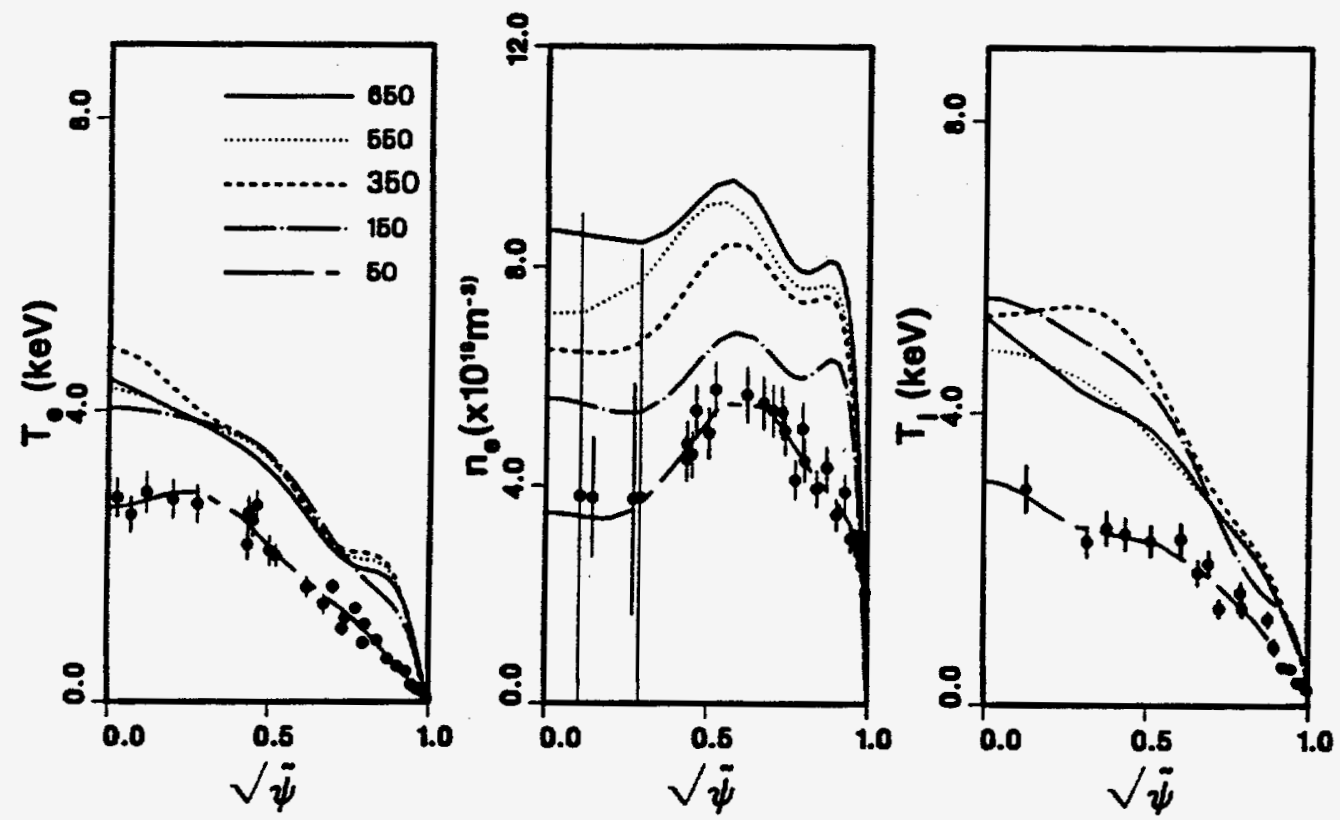

Fig. 7. Temperature and density profile evolution in an $\mathrm{H}$-mode plasma vs. $\sqrt{\tilde{\psi}}$. Shot 80072, $I_{p}=2 \mathrm{MA}, P_{b}=10 \mathrm{MW}$, SHAPE $C, q_{95}=3.4$. Times are in $\mathrm{ms}$, relative to the start of beam-heating. 
and input power (10 MW). Shot 79604 has the highest $\beta \cdot \tau_{E}$ achieved on DIII-D, reaching $\beta \tau_{E}=1.9 \% s\left(\beta \tau_{E}^{\text {th }}=1.7 \% s\right)$.

The central four points on the density plot are from a visible bremsstrahlung diagnostic and are generated using an assumption that $Z_{\text {eff }}$ is a flux surface quantity, constant within the region from the magnetic axis to the vertical Thomson chord. In Fig. 7 we show the uncertainty in this calculation of point values from chordal measurements. However, when data is available for $\bar{n}_{e}$ from a midplane interferometer, the results of this calculation are consistent with the measurement.

The primary feature in the difference between low and high triangularity plasmas lies in the efficiency of central heating as shown these figures. The difference in $T_{e}$ and $T_{i}$ profiles shown here is typical of the differences between $\mathrm{H}$-mode and $\mathrm{VH}$-mode in DIII-D; namely the strong temperature gradient within the region $\sqrt{\bar{\psi}} \lesssim 0.8$ for high triangularity.

The observation that central temperatures and $q_{0}$ both rise in the high performance plasmas motivates us to study the evolution of the discharge more thoroughly. To gain a better understanding of the factors influencing the evolution of the current profile, particularly in the center of the discharge the time evolution of Shot 78136 has been modeled with the WHIST transport code [15]. We return to 78136 because the quality of MSE data gives us greater confidence in our knowledge of the q-profile. Additionally, for this plasma there is lineaveraged density data from a midplane interferometer, reducing the uncertainty in the density profile.

These simulations confirm that the radial profile of the noninductive current source (bootstrap current plus co-injected neutral beam current) has a positive gradient near the axis (i.e., hollow profile, as seen in Fig. 8 where we show the time evolution of $\left\langle J_{\text {tot }}\right\rangle,\left\langle J_{\text {beam }}\right\rangle$, and $\left.\left\langle J_{\text {boot }}\right\rangle\right)$. This initially drives $J_{0}$ down after beam turn-on. That is, the gradient in the EMF response to the current source is negative as can be seen from combining Faraday's and Ohm's laws, which can be written in flux coordinate representation as [16]:

$$
\frac{\partial \tilde{B}_{p}}{\partial t}=\frac{\partial}{\partial \rho}\left[\eta_{\|}\left(\tilde{J}_{t}-\tilde{J}_{s}\right)\right]
$$

where $\tilde{B}_{p} \equiv \mu_{0} I_{t}(\rho) V^{\prime}(\rho) /(2 \pi)^{2} R_{0}\left\langle g_{\theta \theta} / g\right\rangle$ is an effective poloidal field, $I_{t}(\rho)$ is the toroidal current enclosed by flux surface $\rho, V(\rho)$ is the volume bounded by $\rho$ and prime denotes $\partial / \partial \rho, g$ is the Jacobian of the transformation from cylindrical to curvilinear coordinates, $\tilde{J}_{a} \equiv\left\langle\vec{J}_{a} \cdot \vec{B}\right\rangle / \mu_{0} F(\rho) R_{0}\left\langle 1 / R^{2}\right\rangle$ (the subscript $a$ represents either the source current $s$ or the total current $t)$ is an effective current density and $F(\rho)$ is the poloidal current external to surface $\rho$.

Prior to beam turn-on at $2 \mathrm{~s}$ the current profile is nearly in Ohmic equilibrium at the center because of sawtooth activity so $\eta_{\|} \tilde{J}_{t} \approx$ const. Immediately after beam turn-on the slightly hollow current source term from the beam dominates soon to be reinforced by the bootstrap current, causing $\tilde{B}_{p}$ (and $\tilde{J}_{t}$ ) to decrease and $q_{0}$ to increase. At $2250 \mathrm{~ms}$ the beam and bootstrap contributions to the central current density have become approximately equal and represent half the total current. Beam penetration decreases as the discharge progresses because of the density rise and causes the gradients in both the beam and bootstrap currents to relax. The simulations show the $\approx 50 \mathrm{~ms}$ time scale for $q_{0}$ to 

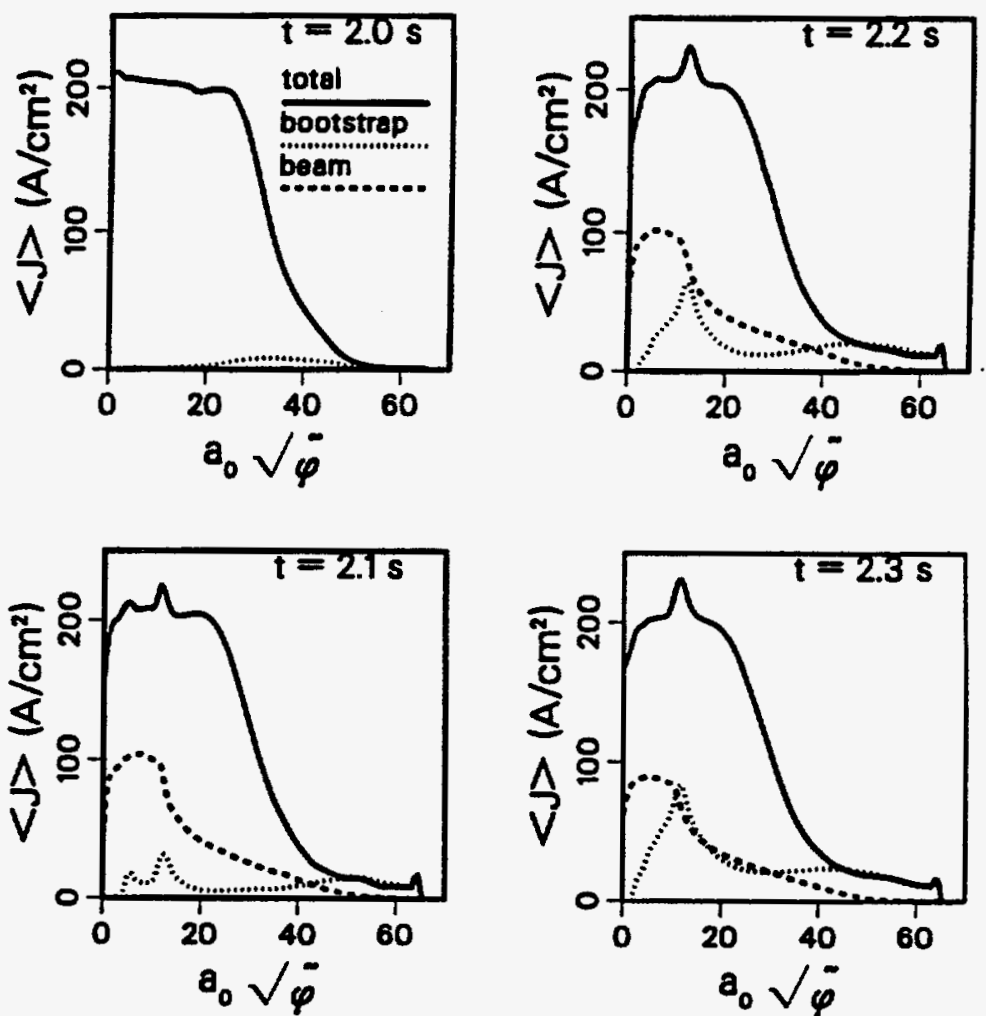

Fig. 8. The time evolution of flux surface averaged current density vs. minor radius times square root of normalized toroidal flux, $a_{0} \sqrt{\phi / \phi_{b d r y}}(\mathrm{~cm})$ Total $J$ as well as bootstrap and neutral beam contributions are shown.

rise and the $\approx 300 \mathrm{~ms}$ time scale for it to fall, using neoclassical resistivity [17] and the bootstrap current expressions of Hirshman [18]. The amount of rise in $q_{0}$ is very sensitive to the beam deposition profile and would therefore be expected to result in variability from shot to shot as the density varies. As the bootstrap current rises, $\kappa_{0}$ rises, contributing to the rise in $q_{0}$. The simulation shows $q_{0}>1$ lasting about $0.5 \mathrm{~s}$ and indicates the broadening beam deposition profile as responsible for the return of $q_{0}$ to unity as both the NBCD and bootstrap contributions fade away. This is also consistent with the experiment which shows longer $q_{0}>1$ intervals and lower maxima for $q_{0}$ with slower rises in the density which occur when the beam power is reduced.

The measured electron and ion temperature and the electron density profiles are well represented by a combination of neoclassical plus anomalous transport with $D^{\text {an }}=\chi_{i}^{\text {an }}=\chi_{e}^{\text {an }}=0.12\left[1+4(\rho / a)^{2}\right] \mathrm{m} / \mathrm{s}^{2}$. Within the half-radius the diffusivity is $3-4$ times the ion neoclassical value. The dominant loss is ion heat conduction. The simulations also reproduce the evolution of the density profile during beam heating. The local peaks at the center and edge at $2.3 \mathrm{~s}$ reflect the beam source and influx from the walls respectively, combined with relatively weak diffusivity.

Shot 80072 (Fig. 7) begins ELMing and sawtoothing about $200 \mathrm{~ms}$ after beam heating begins. It also develops an $\mathrm{m} / \mathrm{n}=3 / 2$ mode at about this time. Until this time there is no significant difference in heating rates. $q_{0}$ may initially 
rise to a value slightly above unity, but remains below the value of about 1.12 required to satisfy the Mercier criterion.

Taken together, and noting that the same $q_{0}$ behavior is observed throughout the experiment, we conclude that the development of the bootstrap current is critical to the rise in $q_{0}$. Initially the beam heating will result in a decreased $J_{0}$ leading to a slight rise in central $q_{0}$. If the plasma. satisfies the Mercier criterion, central (negative) $p^{\prime}$ builds, the bootstrap current conrtributes to a rise in the current density off-axis, higher $\kappa_{0}$, and another source term with a positive gradient at the axis. All serve to sustain the increase in $q_{0}$ which reaches a value well-above unity. Where the Mercier criterion is not satisfied, central $p^{\prime}$ does not build sufficiently and $q_{0} \lesssim 1$ is observed. The plasma remains in $\mathrm{H}$-mode.

\section{STABILITY}

We begin this section with a few general observations on the characteristics of the plasmas discussed here. These remarks refer to the beam-heated plasma in it's VH-mode phase, but neither the Ohmic target nor the ELMing $\mathrm{H}$-mode phases. First, the most prominent theoretical difference between shapes is that the high $\delta$ plasmas satisfy the Mercier criterion (with negative $p^{\prime}$ ) in either its ideal or resistive version [19], while the low $\delta$ plasmas do not. Second, the magnetic well, $W=\frac{V}{\left\langle B^{2}\right\rangle} \frac{d}{d V}\left\langle\mu_{0} p+B^{2} / 2\right\rangle$ is about eight times deeper for the high $\delta$ plasmas. Conceptually, the depth of the magnetic well represents the minimum amount of energy gained by a unit volume of plasma to escape the relevant confinement region. A deep magnetic well therefore represents an energetically stable plasma. Similarly. if plasma confinement is spoiled by plasma turbulence, with comparable level of turbulence, we would also expect the plasma confinement to improve with the depth of magnetic well of a configuration. Third, while we would like to address the question of relative importance of Mercier stability and second stability to ballooning modes, it is the nature of this experiment that they are not separable. Shear will always be reduced in the core plasma by the profile evolution mechanisms described in the prevoius section, thus the attainment of Mercier stability and second-stable access will be coincident.

To illustrate these points we examine the stability of shot 78136 (Fig. 1) in detail. This is a particularly interesting shot reaching the highest triple product on DIII-D, $n_{D}(0) T_{i}(0) \tau_{E}=5 \times 10^{20} \mathrm{~s} \cdot \mathrm{keV} \cdot \mathrm{m}^{-3}$ and $n_{D}(0) T_{i}(0) \tau_{E}^{\text {th }}=$ $4 \times 10^{20} \mathrm{~s} \cdot \mathrm{keV} \cdot \mathrm{m}^{-3}$ at $2290 \mathrm{~ms}$. Although $\beta \cdot \tau_{E}$ continues to rise after this time, the central ion temperature has abruptly collapsed from its peak value of $21 \mathrm{keV}$ to about $14 \mathrm{keV}$. The X-event then occurs at $2430 \mathrm{ras}$ whereupon the plasma reverts to an ELMing $\mathrm{H}$-mode.

The stability of this discharge is as follows: shortly after the start of beam heating $q_{0}$ rises above unity. The dynamics of this process were described in the previous section. The center can support a negative $p^{\prime}$ and remain Mercier stable. The central region will have shear reduced from the already low value on the Ohmic plasma and thus, as soon as the Mercier criterion is satisfied the central region has access to the second regime. The pressure gradient $p^{\prime}$ increases (negatively) as the temperature gradients are increased while the density profile remains flattish. In 78136 , as $q_{0}$ is decreasing an internal $\mathrm{m} / \mathrm{n}=1 / 1$ bursting mode appears during the temperature rise at about the time of the collapse of $T_{i}(0)$ at $2290 \mathrm{~ms}$. As $q_{0}$ continues to fall, the X-event terminates the VH phase at $2430 \mathrm{~ms}$. 
We evaluate stability at $2275 \mathrm{~ms}$, the temperature data being obtained in the interval $2260-2280 \mathrm{~ms}$. This is the latest time for which we obtain a complete data set. The equilibrium is the result of fitting the external magnetics, the pressure profile, and the MSE data ( $q$-profile). The best fit gives a monotonic q-profile with $q_{0}=1.18$. The pressure profile is quite peaked, $p_{0} /\langle p\rangle=4.2$. Ballooning stability is evaluated with the CAMINO [20] code. A composite $s-\alpha$ diagram is obtained by scaling each flux surface to normalize the instability boundary and is shown in Fig. 9 . In the center $(\sqrt{\tilde{\psi}}<0.4)$ most on the plasma remains in the connection region with a few surfaces limited to be above critical values of $\bar{\alpha}$. Prior to this time, this region lies below the stability boundary. The shaded region represents the fact that there is not a universal boundary curve. None of these surfaces actually enters the unstable region. A few surfaces at the outside $(0.90<\sqrt{\tilde{\psi}}<0.94)$ are ballooning unstable. This may reflect the uncertainty in the equilibrium reconstruction; no ELMs occur at this time. $n=1$ stability, evaluated with GATO [21], shows no instability at this time. As can be seen in Fig 1(c), $q_{0}$ has peaked and is decreasing at this time. If we recalculate the equilibrium, reducing $q_{0}$ (by fiat) to 1.10 , we find the equilibrium then to be unstable to a $\mathrm{m} / \mathrm{n}=1 / 1$ "quasi-interchange" mode [22]. Note that there is still no $q=1$ surface in the plasma. This is in excellent agreement with the experimental result, where such a mode is seen at this time. On the soft X-rays we observe a decrease in the rate of rise, but no indication of a sawtooth. They set in at the X-event where $q_{0} \lesssim 1$ [Fig. 10(a)].

Shot 80072, a good example of shape C, with profiles shown in Fig. 7, is a good example of the low $\delta$ cases. The initial behavior is not dissimilar from that described above. $q_{0}$ does rise a bit when beam-heating begins, and may slightly exceed unity. The central $1 / 3$ of the plasma remains Mercier unstable. The central pressure profile remains flat, no bootstrap contribution to the current profile develops and the discharge remains a sawtoothing ELMing $\mathrm{H}$-mode. Note that, were any bootstrap current to evolve, $\kappa_{0}$ would increase, which would further increase the central q values required for Mercier stability.

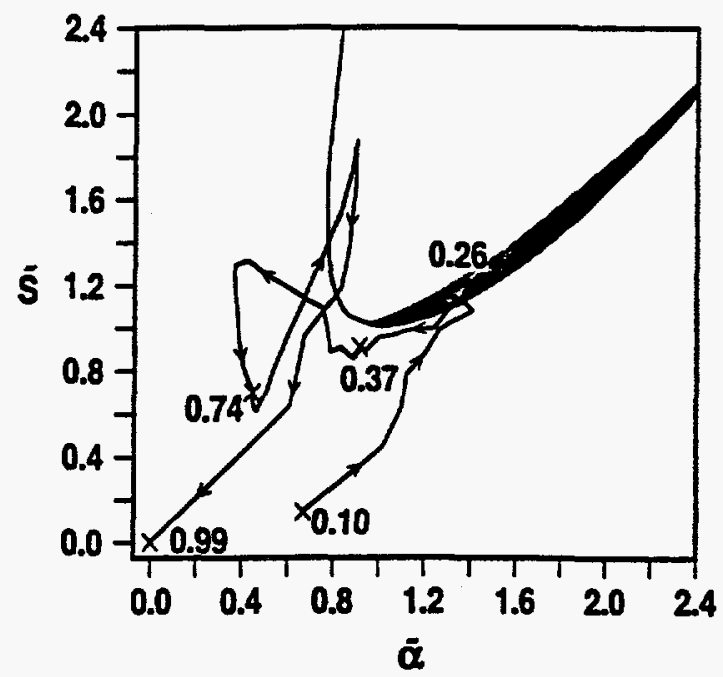

Fig. 9. The normalized $S-\alpha$ diagram for shot 78136 at $2275 \mathrm{~ms}$. The normalization of the experimental points differs for each surface in order to approximate the entire plasma by a single instability boundary. Labels are $\sqrt{\bar{\psi}}$. 

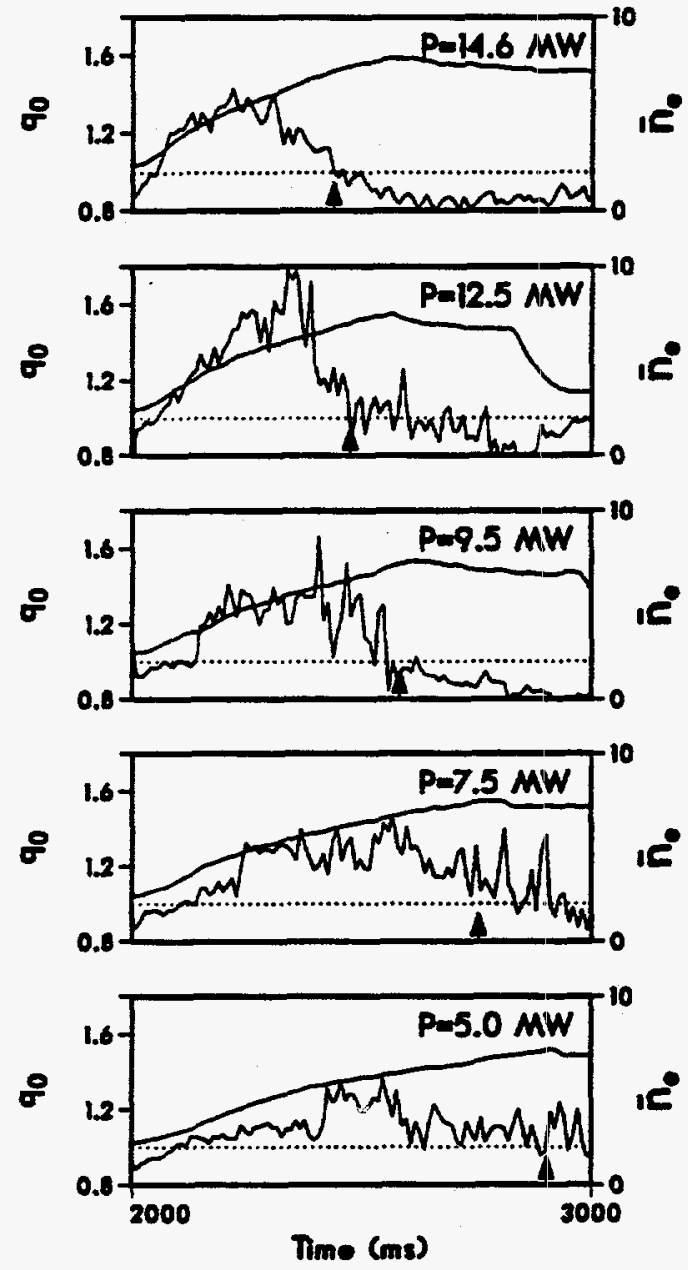

Fig. 10. VH-mode development tor varying beam power. As beam power is increased, denisty rises faster and the VH-phase terminates earlier. The arrow indicates the time at which the plasma is again in $H$-mode. The termination correlates with $q_{0}$ returning to unity. $q_{0}$ is obtained from equilibrium fits with MSE data at $10 \mathrm{~ms}$ intervals. $\bar{n}_{e}$ in units of $10^{19} \mathrm{~m}^{-3}$.

\section{SUMMARY AND DISCUSSION}

We have completed an experiment to compare plasma performance in four shapes in ELM-free, non-sawtoothing VH-mode plasmas. Despite our best effort, such plasmas are not observed when $\delta$ is low. For high triangularity cases, at both $\kappa=2.1$ and $\kappa=1.7$ we obtain $\mathrm{VH}$-mode. The duration is considerably longer at the higher elongation. In the course of the experiment a new record triple product of $n_{D}(0) T_{i}(0) \tau_{E}=5 \times 10^{20} \mathrm{~s} \cdot \mathrm{keV} \cdot \mathrm{m}^{-3}$ at $T_{i}(0)$ in excess of $20 \mathrm{keV}$ was achieved and a new record $\beta \cdot \tau_{E}$ of $1.9 \% . s$, the former under conditions $T_{i} \gg T_{e}$ and the latter with $T_{i} \approx T_{e}$. In both cases the fast ion contribution to the total stored energy was small.

We find that plasma shape is an important consideration, consistent with previous DIII-D results. One aspect of this dependence is simply the change in 
the ratio $I_{p} / q_{\psi}$ with plasma geometry. The importance of this ratio arises from the observed degradation in confinement time as $q_{95}$ is decreased below 5 .

Aside from this ratio, we find further improvement with higher triangularity of up to $50 \%$ in $\beta \cdot \tau_{E}$. The primary experimental signature of such an improvement is improved central heating. This improvement is correlated with the satisfying the Mercier criterion and the consequent access to the second-stable regime in the plasma core. We find performance may be somewhat limited by the onset of the $\mathrm{m} / \mathrm{n}=1 / 1$ quasi-interchange internal mode. Thus far in our investigation we nave not observed values of $\alpha$ more than a factor of two beyond the first-stable boundary.

The role played by the Mercier stable core in VH-mode is not completely clear. In poorly shaped plasmas which do not achieve Mercier stability the ELMfree sawtooth-free period is short. However, up to the time of the first ELM the pressure profiles evolve similarly for both the good and poor shapes. After ELMs and sawteeth onset the strong central peaking of the pressure profile is not observed. This distinction is consistent with the second-stable access. Discharges with long ELM-free periods but without $\mathrm{VH}$-mode have been observed at low triangularity and low power, however long ELM-free sawtooth-free periods have not been observed at higher power without central Mercier stability.

Because of volume effects, improvements on the central region do not contribute a large effect on $\tau_{E}$. A large part of the confinement improvement in $\mathrm{VH}$-mode occurs in a region outside that which is effected by Mercier stability. Also, other work [9], shows a good corelation between increased shear in the $\vec{E} \times \vec{B}$ flow velocity, reduced density fluctuation levels, improved heating effciency in the region $\sqrt{\bar{\psi}} \gtrsim 0.8$, and increased confinement time. An understanding of the relationship of the improved core performance with the improvments in the outer region remains a challenge.

Although we do not have space to discuss the termination of the VH-phase (the "X-event"), it is noteworthy that the X-event occurs at the time when $q_{0}$ returns to approximately unity. With time resolution of about $10 \mathrm{~ms}$ we cannot determine whether $q_{0}$ returning to unity occurs before or after the X-event. It is clear that $q_{0}$ begins its decrease well before the termination. As beam power is increased (Fig. 10), we observe a higher initial rise in $q_{0}$, and a faster return to unity. The density rise is also faster with increased beam power, and of course, the VH-phase is shorter. These features are consistent with our hypothesis of $q_{0}$ driven by beam and bootstrap current sources.

As stated, one motivation of the experiment was the determination of a shape consistent with the DIII-D program goals, which is shape B. Having modelled 78136 so successfully, it is interesting to proceed a bit further and inquire as to the evolution of such a plasma with particle control. If we were to pump 78136 well enough to maintain $\bar{n}_{e}=7 \times 10^{19} / \mathrm{m}^{3}$, but other than that make no change in the modelling of 78136 , the results are most encouraging. WHIST predicts the discharge would be maintained near peak conditions with $T_{i}(0) \approx 25 \mathrm{keV}$, $T_{e}(0) \approx 9 \mathrm{keV}$, and $n_{e}(0) \approx 9 \times 10^{19} / \mathrm{m}^{3}$. With the density peaking eventually brought about by the beam fueling of the core, such a discharge might exhibit a more substantial occupation of the second-regime of ballooning stability. Under this scenario, $88 \%$ of the current is non-inductive, asymptotically, $0.9 \mathrm{MA}$ from bootstrap and 0.5 MA from NBCD. The evolution shows throughout the $5 \mathrm{~s}$ simulation,after thermal and particle equilibrium have been reached, a slowly decreasing $q_{0}$, from 1.4 after the initial transients to 1.1 at the end of the beam pulse. On balance, the result is extremely encouraging for the future DIII-D 
program. With RF current drive, it seems plasuible that such a discharge could be maintained indefinately [2].

\section{REFERENCES}

[1] ALLEN, S.L., et al., in Proc. 11th International Conference on Plasma Surface Interactions in Controlled Fusion Devices, (Ibaraki-ken, Japan, 1994) to be published.

[2] TAYLOR, T.S., in Proc. 21st European Conferene on Contr. Fusion and Plasma Physics, Montpellier, France 1994 (European Physical Society, Petit-Lancy, Switzerland, to be published).

[3] LAZARUS, E.A., in Proc. 20th European Conferene on Contr. Fusion and Plasma Physics, Lisbon, Portugal (European Physical Society, PetitLancy, Switzerland, 1993), Vol. 17C, Part I, p. 95.

[4] TODD, T.N., and D.C. ROBINSON, Phys. Rev. Lett. 48, 1359 (1982).

[5] LAZARUS, E.A., et al., Phys. Fluids B 4, 3644 (1992).

[6] TROYON, F., GRUBER, R., SAUERENMANN, H., SEMENZATO, S., SUCCI, S., Plasma Phys. Contr. Fusion bf 26, 209 (1984).

[7] LAZARUS, E.A., et al., Phys. Fluids B 3, 2220 (1991).

[8] JACKSON, G.L., et al., Phys. Rev. Lett. 67, 3098 (1991).

[9] OSBORNE, T.H.,et al., Nucl. Fusion (to be published).

[10] STRAIT, et al., in Proc. 20th European Conferene on Contr. Fusion and Plasma Physics, Lisbon, Portugal (European Physical Society, Petit-Lancy, Switzerland, 1993), Vol. 17C, Part I, p. 211.

[11] HYATT, A.W., et al., in Proc. 11th International Conference on Plasma Surface Interactions in Controlled Fusion Devices, (Ibaraki-ken, Japan, 1994) to be published.

[12] SCHISSEL, D.P., et al., Nucl. Fusion 31, 73 (1991).

[13] STAMBAUGH, R.D., S.L. ALLEN, N.H. BROOKS, D.N. HILL, D.L. HILLIS, et al., Plasma Phys. Contr. Fusion 36249 (1994).

[14] YUSHMANOV, P.N., et al., Nucl. Fusion 30, 1999 (1990).

[15] HOULBERG, W.A., S.E. ATTENBERGER, L.M. HIVELY, Nucl. Fusion 32, 935 (1982).

[16] HOULBERG, W.A., Nucl. Fusion $\mathbf{3} 371009$ (1987).

[17] HINTON, F.L., R.D. HAZELTINE, Rev. Modern Phys. 48, 239 (1976).

[18] HIRSHMAN, S.P., Phys. Fluids 31, 3150 (1988).

[19] GLASSER, A.H., J.M. GREENE, J.L. JOHNSON, Phys. Fluids 18, 875 (1975).

[20] CHANCE, M.S., in Proc. Theory of Fusion Plasma, (Varenna, 1987) Vol. 18 , p. 87.

[21] BERNARD, L.C., F.J. HELTON, R.W. MOORE, Comput. Phys. Commun. 24, 377 (1981).

[22] WAELBROECK, F.L., R.D. HAZELTINE, Phys. Fluids 31, 1217 (1988).

\section{ACKNOWLEDGMENTS}

This is a report of work supported by the U.S. Department of Energy under Contract Nos. DE-AC03-89ER51114, DE-AC05-84OR21400, W-7405-ENG-48, DE-AC04-94AL85000, and Grant Nos. DE-FG03-89ER51121 and DE-FG03$86 \mathrm{ER} 53225$. 